\title{
Análisis Exergético de la Gasificación de Biomasa
}

\author{
Jorge M. Mendoza ${ }^{(1,2)}$, Antonio J. Bula ${ }^{(2)}$, Rafael D. Gómez ${ }^{(1)}$ y Lesmes A. Corredor ${ }^{(2)}$ \\ (1) Universidad Pontificia Bolivariana, Facultad de Ingeniería Mecánica, km 8 Vía Cereté, \\ Montería-Colombia ( e-mail: jorge.mendoza@upb.edu.co, rafael.gomezv@upb.edu.co), \\ (2) Universidad del Norte, km 5 Antigua Vía Puerto Colombia, Barranquilla-Colombia, \\ Departamento de Ingeniería Mecánica, (abula@uninorte.edu.co, Icorredo@uninorte.edu.co)
}

Recibido Dic. 26, 2011; Aceptado Feb. 07, 2012; Versión final recibida Mar. 27, 2012

\begin{abstract}
Resumen
El objetivo de este estudio fue realizar el análisis exergético de la gasificación de biomasa residual con la finalidad de obtener energía útil. Se desarrolló un modelo para la gasificación de biomasa basado en el equilibrio químico de las reacciones. Este modelo permite predecir la evolución de la composición del gas de síntesis en función de la temperatura, las presiones y la composición elemental de los residuos del proceso de extracción de aceite de palma y orujo de lavado de uva (hidrogeno, oxigeno, carbono y nitrógeno), como también hacer un análisis exergético. Se encontró que independiente del agente gasificante, la concentración de monóxido de carbono e hidrógeno tienden a incrementar significativamente a altas presiones y elevadas temperaturas. Además, se muestra que la eficiencia exergética incrementa con la temperatura y disminuye con el aumento de la relación aire/combustible.
\end{abstract}

Palabras clave: biomasa, gasificación, equilibrio químico, exergía

\section{Exergy Analysis of Biomass Gasification}

\begin{abstract}
This paper shows the exergy analysis applied to the gasification process of residual biomass with the purpose of obtaining useful energy. A model for the biomass gasification based on chemical equilibrium of the reactions is proposed. The model allows predicting the syngas composition as a function of temperature, pressure and ultimate analysis of palm oil and grapevine pruning waste (determining hydrogen, oxygen, carbon and nitrogen). It was found that the carbon monoxide and hydrogen concentration significantly increase at high pressure and temperature independently of the gasifying agent. Furthermore, it was also noticed that the exergy efficiency increases as temperature increases, and decreases as the relative fuel/air ratio increases.
\end{abstract}

Keywords: biomass, gasification, chemical equilibrium, exergy 


\section{INTRODUCCIÓN}

Los residuos de la palma africana, como cualquier otra biomasa de tipo vegetal, está constituida por materia orgánica que tuvo origen inmediato en un proceso biológico como lo es la fotosíntesis. Este residuo agrícola puede emplearse como combustible debido a la presencia de moléculas celulósicas que contienen energía en sus enlaces y que se libera bajo condiciones de oxidación total o parcial. Los residuos de la palma africana contienen un rango del 20.8-59.7 de celulosa, aportando por unidad de masa, más de la mitad de la energía contenida en un combustible como el carbón (Chew y Bhatia, 2008; Abdullaah y Gerhauser, 2008; Kelly et al., 2007). Toda la biomasa proveniente de la palma africana es rica en lignina, celulosa y hemicelulosa, las cuales actualmente representan grandes beneficios en la producción de vapor para el proceso de obtención de aceite vegetal. En los último años la gasificación de biomasa vegetal se ha venido convirtiendo en una importante alternativa para países del tercer mundo que dependen de combustibles fósiles como el carbón y el petróleo, emergiendo como una tecnología prometedora para suplir la necesidad de un suministro de energía limpia y descentraliza, mientras que el orujo lavado de uva (orujo desalcoholizado, orujo agotado u orujillo de uva) se obtiene a partir del orujo de uva (hollejos, resto de raspones y granilla) que se genera en las prensas de las bodegas. La masa que queda tras este proceso es lo que se denomina orujo lavado de uva u orujo desalcoholizado u orujo agotado. Este orujo desalcoholizado tiene una humedad considerable, entre el $55 \%$ y $75 \%$, pero suele ser secado en las propias alcoholeras quedándose con un $15 \%$ de humedad, obteniéndose así un buen combustible (Pazo, 2006).

La gasificación es un proceso donde ocurre una transformación termoquímica en la cual un sustrato carbonoso es transformado en un gas combustible de bajo poder calorífico, a través de una serie de reacciones que ocurren a una temperatura determinada en presencia de un agente gasificante (aire, oxigeno y/o vapor de agua). En la gasificación a diferencia de la combustión se presenta un exceso de combustible sólido lo que provoca una combustión incompleta aumentando la generación de gases combustibles como el monóxido de carbono $(\mathrm{CO})$, hidrogeno $\left(\mathrm{H}_{2}\right)$, trazas de metano $\left(\mathrm{CH}_{4}\right)$ y alquitránes (Lucas et al., 2004). Esta transformación termoquímica según las temperaturas de operación en un gasificador puede generar gas manufacturado con distintas composiciones (Mendoza et al., 2011; Cujia y Bula, 2010; Hernández et al., 2010ª; Hernández et al., $\left.2010^{\mathrm{b}}\right)$. A altas temperaturas la formación de hidrogeno $\left(\mathrm{H}_{2}\right)$ es más rica mientras que la concentración de metano $\left(\mathrm{CH}_{4}\right)$ disminuye (Lucas et al., 2004).A bajas temperaturas se transforma esencialmente en electricidad (Perez, 2007). Adicionalmente la exergía se puede definir como el trabajo máximo que puede obtenerse a partir de un sistema hasta que se alcanza el equilibrio con su entorno" (Kotas, 1985). En comparación con la energía la cual es solo función de los estados de la materia, la Exergía es función de estos estados y de los componentes del ambiente (Olah G. et al., 2006), La exergía química expresa el contenido exergético de una sustancia con referencia a las condiciones de presión y temperatura del ambiente (Mathews, 2007). Este valor resulta del trabajo que puede obtener una sustancia que se encuentra a una presión y temperatura del entorno, si alcanza un estado de equilibrio termodinámico. El análisis exergético consiste en evaluar cada portador de energía en el sistema e identificar su composición química y estado físico.

\section{DISEÑO DEL MODELO FUNCIONAL}

El modelo funcional se genera un proceso de transformación de biomasa a gas pobre, mediante los principios de la Primera Ley, equilibrio termodinámico para las reacciones dadas y el principio de la energía libre de Gibbs. El modelo de gasificación realiza atendiendo las siguientes suposiciones:

- El sistema es un modelo de flujo estacionario.

- Las condiciones de referencia ambiente son $T_{0}=25^{\circ} \mathrm{C}$ y $\mathrm{P}_{0}=1 \mathrm{~atm}$.

- El tiempo de residencia en las reacciones es el suficiente para que se dé en equilibrio.

- La temperatura y presión del gas pobre son las mismas en todo el proceso de gasificación.

- Se emplearon relaciones de gas ideal para las propiedades de los gases. 
- La transferencia de calor y la presión de operación en el proceso de gasificación se asumen constantes.

\begin{tabular}{|c|c|c|c|c|}
\hline \multicolumn{2}{|c|}{ PRIMERA ETAPA } & $\begin{array}{c}\text { CONDICIONES DE OPERACIÓN } \\
\text { PlaAs } \mathrm{T}_{\text {Gas }} \\
+\end{array}$ & \multicolumn{2}{|c|}{ SEGUNDA ETAPA } \\
\hline $\begin{array}{c}\text { BIOMASA } \\
\mathrm{C} \\
\mathrm{H} \\
\mathrm{O} \\
\mathrm{N} \\
\mathrm{W}\end{array}$ & $\begin{array}{c}\text { REACCIONES } \\
\text { HETEROGÉNEAS } \\
\text { C+2 } \mathrm{H}=\mathrm{CH}_{4} \\
\mathrm{c}+\mathrm{H}_{2} \mathrm{O}=\mathrm{CO}+\mathrm{H}_{2} \\
\mathrm{C}+\mathrm{CO}_{2}=2 \mathrm{CO}\end{array}$ & $\begin{array}{c}\text { COMPOSICIÓN } \\
\mathrm{xCO}^{\mathrm{xCO}_{2}} \mathrm{xCH}_{2} \\
\mathrm{xCH}_{4} \mathrm{xH}_{2} \mathrm{O} \mathrm{xN}_{2} \\
\text { Moles de gas } \\
\mathrm{m}_{\mathrm{gas}} \\
\text { EQUIVALENTE RATIO } \\
\text { ER (estequiométrico) }\end{array}$ & $\begin{array}{c}\text { REACCIONES } \\
\text { HOMOGÉNEAS } \\
\mathrm{cO}+\mathrm{H}_{2} \mathrm{O}=\mathrm{CO}_{2}+\mathrm{H}_{2} \\
\mathrm{CO}+3 \mathrm{H}_{2}=\mathrm{CH}_{4}+\mathrm{H}_{2} \mathrm{O}\end{array}$ & $\begin{array}{l}\text { GAS PRODUCTO } \\
\text { Composición molar } \\
y \mathrm{CO}_{\mathrm{yCO}_{2}} \mathrm{yH}_{2} \\
\mathrm{yCH}_{4} \mathrm{yH}_{2} \mathrm{O} \mathrm{yN}_{2} \\
\text { Moles. Gas producto } \\
\mathrm{n}_{\mathrm{zex}}\end{array}$ \\
\hline
\end{tabular}

Fig. 1: Esquema del modelo matemático para la estimación de la composición del gas producto

\section{BALANCE DE MASA}

En la primera etapa del modelo, el carbono solido es convertido en fase gaseosa en presencia de hidrogeno y oxigeno tal como lo muestran las reacciones (1), (2) y (3).

$$
\begin{aligned}
& \mathrm{C}+2 \mathrm{H}=\mathrm{CH}_{4} \\
& \mathrm{C}+\mathrm{H}_{2} \mathrm{O}=\mathrm{CO}+\mathrm{H}_{2} \\
& \mathrm{C}+\mathrm{CO}_{2}=2 \mathrm{CO}
\end{aligned}
$$

La reacción (1) es exotérmica y favorece la formación de metano, la reacción (2) es endotérmica conocida como Water Gas Shift, en la cual el vapor de agua se convierte en hidrogeno mientras se da la reacción 3 para la formación de CO conocida como la reacción de Boudouard.

$$
\begin{aligned}
& \exp \left[\frac{\left(g_{\mathrm{c}}^{*}+2 g_{\mathrm{H}_{2}}^{*}-g_{\mathrm{CH}_{4}}^{*}\right)}{\mathrm{RT}}\right]=\frac{x_{\mathrm{CH}_{4}}}{x_{\mathrm{H}_{2}}^{2}}\left(\frac{P_{0}}{P_{\text {gas }}}\right) \\
& \exp \left[\frac{\left(\mathrm{g}_{\mathrm{c}}^{*}+\mathrm{g}_{\mathrm{H}_{2} \mathrm{O}}^{*}-\mathrm{g}_{\mathrm{CO}}^{*}-\mathrm{g}_{\mathrm{H}_{2}}^{*}\right)}{\mathrm{RT}}\right]=\frac{x_{\mathrm{CO} \boldsymbol{x}_{2}}}{\boldsymbol{x}_{\mathrm{H}_{2} \mathrm{O}}}\left(\frac{\boldsymbol{P}_{\text {gas }}}{\boldsymbol{P}_{0}}\right) \\
& \exp \left[\frac{\left(\mathrm{g}_{\mathrm{c}}^{*}+\mathrm{g}_{\mathrm{CO}_{2}}^{*}-2 \mathrm{~g}_{\mathrm{CO}}^{*}\right)}{\mathrm{RT}}\right]=\frac{\mathrm{x}_{\mathrm{CO}}^{2}}{\mathrm{x}_{\mathrm{CO}_{2}}}\left(\frac{\mathrm{P}_{\mathrm{gas}}}{\mathrm{P}_{0}}\right)
\end{aligned}
$$

Las ecuaciones (4), (5) y (6), describen el equilibrio de las reacciones heterogéneas para e carbón sólido. $\mathrm{P}_{\mathrm{gas}}$ Indica la presión dada en las reacciones, $\boldsymbol{P}_{0}=101.325 \mathrm{kPa}$ la presión estándar de referencia y $\mathrm{T}\left({ }^{\circ} \mathrm{C}\right)$ indica la temperatura de la gasificación. Además de las reacciones del carbono, se presentan también las siguientes reacciones homogéneas.

$$
\begin{aligned}
& \mathrm{CO}+\mathrm{H}_{2} \mathrm{O}=\mathrm{CO}_{2}+\mathrm{H}_{2} \\
& \mathrm{CO}+3 \mathrm{H}_{2}=\mathrm{CH}_{4}+\mathrm{H}_{2} \mathrm{O}
\end{aligned}
$$

El equilibrio termodinámico de las reacciones (7) y (8) se describen en las ecuaciones (9) y (10) respectivamente:

$$
\exp \left[\frac{\left(\mathrm{g}_{\mathrm{CO}}^{*}+\mathrm{g}_{\mathrm{H}_{2} \mathrm{O}}^{*}-g_{\mathrm{CO}_{z}}^{*}-g_{\mathrm{H}_{2}}^{*}\right)}{\mathrm{R} T}\right]=\frac{y_{\mathrm{CO}_{2}} y_{\mathrm{H}_{2}}}{y_{\mathrm{CO} y_{\mathrm{H}_{2} \mathrm{O}}}}
$$




$$
\exp \left[\frac{\left(\mathrm{g}_{\mathrm{Co}}^{*}+3 \mathrm{~g}_{\mathrm{H}_{2}}^{*}-\mathrm{g}_{\mathrm{CH}_{4}}^{*}-\mathrm{g}_{\mathrm{H}_{2} \mathrm{O}}^{*}\right)}{\mathrm{RT}}\right]=\frac{\mathrm{y}_{\mathrm{CH}_{4}} \mathrm{y}_{\mathrm{H}_{2}}}{\mathrm{y}_{\mathrm{CO}} \mathrm{Y}_{\mathrm{H}_{2}}^{3}}\left(\frac{P_{0}}{\boldsymbol{P}_{\text {gas }}}\right)^{2}
$$

La función de Gibbs para cada componente i se determina como lo indica la ecuación (11):

$g_{i}^{*}=\left(g_{f}^{0}\right)_{i}+\left(h_{T}-h_{T_{0}}\right)_{i}-T\left(S_{T}\right)_{i}+T_{0}\left(S_{T_{0}}^{0}\right)_{i}$

Identificados los mecanismos de reacción presentes y los modelos termodinámicos de equilibrio, se realiza un balance de masa tanto para la etapa de reacciones heterogéneas como la etapa de reacciones homogéneas. La ecuación (12) muestra un balance de masa aplicado a las reacciones del carbono solido en la etapa 1, adicionalmente La suma de las fracciones molares de los componentes del gas producto debe sumar la unidad como se muestra en la ecuación (13).

$\mathrm{z}_{C}+\mathrm{z}_{O}+\mathrm{z}_{H}+\mathrm{z}_{\mathbb{N}}+\mathrm{z}_{W}+n_{\mathrm{a}} \mathrm{M}_{\mathrm{a}}+n_{H 20} M_{H 20}=m_{\text {gas }}\left(\sum_{\mathrm{i}} \mathrm{x}_{\mathrm{i}} \mathrm{M}_{\mathrm{i}}\right)+z_{C}$ Char

$\sum_{i} x_{i}=1$

Donde $n_{\alpha}$ Indica las moles de aire usadas en la gasificación de biomasa, la cual está relacionada con el Equivalente Ratio (ER) tal como se muestra en la ecuación (14).

$\mathrm{E} R=\frac{0.21 \mathrm{n}_{\mathrm{a}}}{\mathrm{n}_{\mathrm{C}}+0.5 n_{H}-n_{0}}$

La ecuación (15) muestra el balance de masa aplicado en la segunda etapa, en este paso la composición del gas producto es convertida mediante las reacciones (7) y (8), para determinar la composición final del gas producto.

$\mathrm{m}_{\text {gas }}\left(\sum_{\mathrm{i}} \mathrm{x}_{\mathrm{i}} \mathrm{M}_{\mathrm{i}}\right)=n_{\text {gas }}\left(\sum_{i} y_{i} M_{i}\right)$

\section{BALANCE DE ENERGÍA}

El balance de energía total para la gasificación de biomasa se detalla en la ecuación (16).

$$
L \mathrm{HV}_{\text {biomasa }}+n_{\alpha} \Delta h_{\alpha}+n_{H_{2} \mathrm{O}}\left(h_{f g}+\Delta h_{\mathrm{H}_{2} \mathrm{O}}\right)=\mathrm{m}_{\mathrm{gas}}\left(\Delta \mathrm{h}_{\text {gas }}+\mathrm{LVH} \mathrm{H}_{\text {gas }}\right)+Z_{c} \operatorname{Char}\left(\Delta h_{C}-C_{C}\right)+Q_{1}
$$

$\Delta \mathrm{h}_{\mathrm{a}}, \Delta h_{H_{\mathrm{z}} \mathrm{O}}$ y $\Delta \mathrm{h}_{\mathrm{gas}}$ son las diferencias de entalpias de el aire, el agua y el gas producto evaluados a la temperatura $\mathrm{T}_{\text {gas }}$ y la temperatura de referencia del modelo termodinámico $\mathrm{T}_{0}=25^{\circ} \mathrm{C}$.

$L \mathrm{HV}_{\text {biomasa }}[\mathrm{kJ} / \mathrm{kg}$ ] Es el poder calorífico inferior de la biomasa el cual es determinado con la ecuación (17) desarrollada por (Channiwala y Parikh, 2002) el poder calorífico del gas producto $\mathrm{LVH}_{\mathrm{gas}}[\mathrm{kJ} / \mathrm{kmol}]$ es determinado por la ecuación (18).

$$
\begin{aligned}
& H \mathrm{HV}_{\text {biomasa }}=0.349 \mathrm{z}_{\mathrm{C}}+1.1783 \mathrm{z}_{\mathrm{H}}-0.1034 \mathrm{z}_{0}-0.0151 \mathrm{z}_{N} \\
& \mathrm{HV}_{\text {gas }}=282933 \mathrm{xCO}+802303 \mathrm{xCH}_{4}+241827 x \mathrm{H}_{2}
\end{aligned}
$$

La eficiencia energética del proceso de gasificación se determina con base en las capacidades caloríficas del gas producto y de la biomasa mediante la ecuación (19). 
$\eta=\frac{\mathrm{n}_{\text {gas }} \mathrm{LHV}_{\text {gas }}}{\mathrm{LHV}_{\text {biomasa }}}$

\section{EVALUACIÓN EXERGÉTICA DEL PROCESO.}

La eficiencia exergética, representa la relación entre los flujos de exergía de entrada y de salida del proceso de gasificación en este caso. Las eficiencias exergéticas pueden ser cuantificadas a partir de solo su potencial químico o cuantificando la exergía física. Las ecuaciones (20) y (21) indican la evaluación de estas eficiencias química y total de flujo, la exergía de la biomasa en la ecuación (22) y $\boldsymbol{\beta}$ de la ecuación (23) y (24).

$$
\begin{aligned}
& \Psi_{C H}=\frac{n_{\text {gas }} e_{\text {ch, gas }}}{e_{\text {ch,biomana }}} \\
& \psi=\frac{n_{\text {gas }}\left(e_{\text {ch,gas }}+e_{\text {ph,gas }}\right)}{e_{\text {ch,biomana }}+n_{\text {air }} e_{\text {air }}} \\
& e_{\text {ch,biomasa }}=\beta\left(L H V_{\text {Biomass }}\right) \\
& \beta=\frac{\left(1.0414+0.177\left(\frac{C}{H}\right)-0.3328\left(\frac{O}{\mathrm{C}}\right)(1+0.3377(H))\right)}{1-0.4021\left(\frac{O}{C}\right)}
\end{aligned}
$$

La ecuación (24) determina la exergía física de flujo para gases. Esta relación es usada para determinar la exergía física del gas producto como la exergía física del aire empleado como agente oxidante.

$\mathrm{e}_{\mathrm{ph}}=\left(\mathrm{h}-h_{0}\right)-T_{\mathrm{o}}\left(s-s_{0}\right)$

\section{RESULTADOS Y DISCUSIÓN}

Para el modelo en equilibrio termoquímico y el desarrollado de la evaluación exergética de los residuos de la palma y el orujo lavado de uva, cuyo análisis elemental es mostrado en la tabla 1.

Tabla 1: Análisis elemental de la biomasa modelada

\begin{tabular}{|l|c|c|c|c|c|}
\hline \multirow{2}{*}{ Biomasa } & \multicolumn{4}{|c|}{ Elementos (wt \% base seca) } \\
\cline { 2 - 6 } & $\mathrm{C}$ & $\mathrm{H}$ & $\mathrm{N}$ & $\mathrm{S}$ & $\mathrm{O}$ \\
\hline Fibra y Cuesco promedio & 50.27 & 7.07 & 0.42 & 0.63 & 36.28 \\
\hline Orujo lavado de uva & 47.21 & 5.97 & 2.29 & 0.15 & 37.92 \\
\hline
\end{tabular}

De las figuras $2 a$ y $2 b$ se puede apreciar que la concentración de los gases productos de la gasificación para los dos tipos de biomasa son similares, esto debido a que los análisis últimos son similares.

Para el metano, la generación se ve favorecida con la gasificación a alta presión, obteniéndose para los dos tipos de biomasa valores de fracción molar de metano hasta cuatro (4) veces más altos a presión de 10 bares $\left(0.12\right.$ a $600^{\circ} \mathrm{C}, 0.04$ a $\left.1100^{\circ} \mathrm{C}\right)$ que a 1 bar $\left(0.03\right.$ a $600^{\circ} \mathrm{C}, 0$ a $\left.1100^{\circ} \mathrm{C}\right)$. Desde el punto de vista de la temperatura, para los dos tipos de biomasa e independientemente de las presiones analizadas, la concentración de metano disminuye al aumentar la temperatura, llegando a ser nula la generación a temperaturas mayores a $1000^{\circ} \mathrm{C}$. 


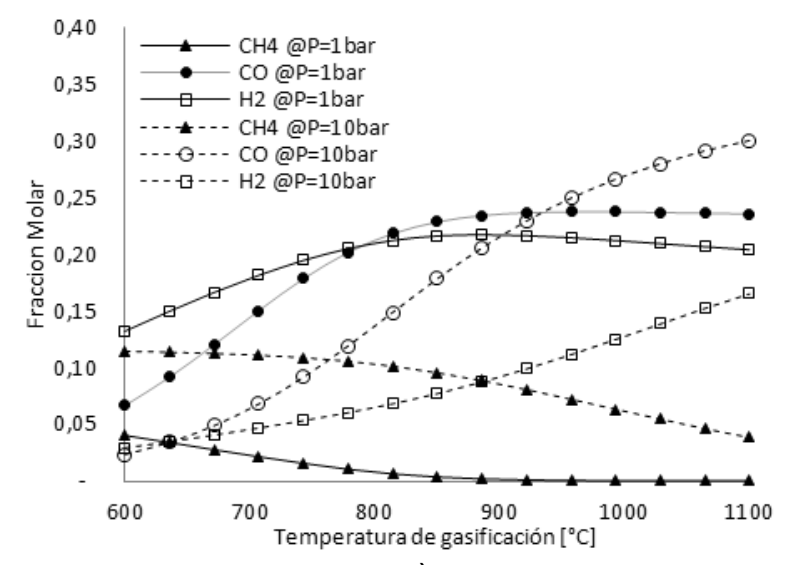

a)

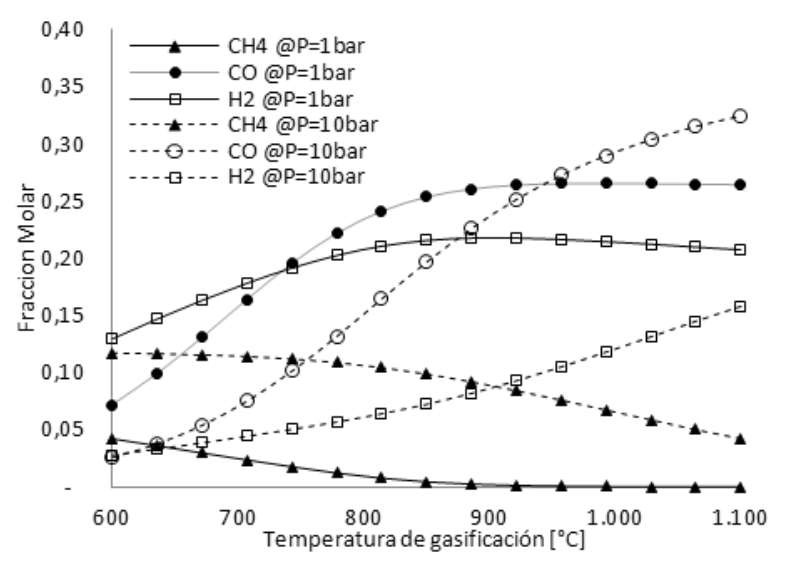

b)

Fig. 2. Variación de la composición de gas producto en función de la temperatura a presiones de 1 bar y 10 bares a) de los residuos de la palma africana y b) el orujo lavado de uva.

En el caso del monóxido de carbono, se aprecia que la presión y la temperatura afectan la concentración obtenida de forma diferente. A alta presión (10 bares), se observa que la concentración del monóxido se incrementa al aumentar la temperatura, sin embargo, este fenómeno se aprecia más para valores de temperatura entre $700^{\circ} \mathrm{C}$ hasta $950^{\circ} \mathrm{C}$, cuando la fracción molar pasa de 0.07 a $0.25\left(7.2 \times 10^{-4} /{ }^{\circ} \mathrm{C}\right)$. Para los valores de temperatura simulados, entre $600^{\circ} \mathrm{C}$ a $700^{\circ} \mathrm{C}$, y $950^{\circ} \mathrm{C}$ a $1100^{\circ} \mathrm{C}$, el incremento de la fracción molar es menor $\left(5 \times 10^{-4} /{ }^{\circ} \mathrm{C}\right)$ y $\left(3.3 \times 10^{-4} /{ }^{\circ} \mathrm{C}\right)$ respectivamente. Para condiciones de baja presión ( 1 bar), se observa que el monóxido de carbono incrementa su concentración de manera apreciable cuando la temperatura varia de $600^{\circ} \mathrm{C}$ hasta $950^{\circ} \mathrm{C}$, pasando la fracción molar de 0.06 a $0.24\left(6.3 \times 10^{-4} /{ }^{\circ} \mathrm{C}\right)$, de allí en adelante la fracción molar permanece casi constante en 0.24 . Desde el punto de vista de la presión, se observa que la fracción molar es mayor a baja presión cuando la temperatura es menor de $950^{\circ} \mathrm{C}$. Para temperaturas mayores a $950^{\circ} \mathrm{C}$, se produce inversión en la tendencia, y se observa que la fracción molar del monóxido continua incrementándose para la presión de 10 bares mientras que para la presión de 1 bar la fracción molar permanece casi constante. A $950^{\circ} \mathrm{C}$, para los dos valores de presión simulados, la fracción molar es de aproximadamente 0.24.

Observado el hidrógeno a condición de baja presión ( 1 bar), entre $600^{\circ} \mathrm{C}$ y $850^{\circ} \mathrm{C}$, se nota que la fracción molar aumenta de 0.12 a 0.22 , aproximadamente. Para temperaturas entre $850^{\circ} \mathrm{C}$ y $1100^{\circ} \mathrm{C}$, la fracción de molar disminuye muy suavemente pasando de 0.22 a 0.20 . Este comportamiento indica que existe un máximo en la generación de hidrógeno, por lo menos para la condición de 1 bar. Para la presión de 10 bares se observa que la fracción molar aumenta de manera continua al incrementarse la temperatura, pasando de 0.03 a $600^{\circ} \mathrm{C}$, hasta 0.16 a $1100^{\circ} \mathrm{C}$. Independientemente del comportamiento, se nota que para el rango de presión y temperatura analizadas, las fracciones molares a baja presión siempre son mayores que a alta presión, acercándose cuando se incrementa la temperatura. Se puede asumir que habrá algún punto de mayor temperatura en el cual se inviertan los valores, como en el caso del monóxido de carbono, pero no se observa debido a la limitante en el rango de temperatura analizado.

Los resultados obtenidos fueron comparados con los datos mostrados por Lucas et al. (2004) para gasificación de biomasa alimentada con aire precalentado a $350^{\circ} \mathrm{C}$ y presión atmosférica, en donde se muestran tendencias similares. Para el monóxido de carbono e hidrógeno, se aprecia que al aumentar la temperatura entre $600^{\circ} \mathrm{C}$ y $1100^{\circ} \mathrm{C}$, la fracción molar de estas especies pasa de 0.15 a 30 y de 0.05 a 0.11 respectivamente. Observándose que en ambas, a temperaturas cercanas a $800^{\circ} \mathrm{C}$ hasta $1100^{\circ} \mathrm{C}$ se alcanza un valor casi constante. Este comportamiento es similar al descrito para el monóxido de carbono e hidrógeno en la presente investigación. Para el metano, se observa que al aumentar la temperatura la fracción molar se hace cero, iniciando en 0.04. Para Lucas et al. (2004) esta fracción molar nula se da alrededor de la máxima temperatura, $1100^{\circ} \mathrm{C}$, mientras que en la presente investigación este valor de temperatura es de $1000^{\circ} \mathrm{C}$ aproximadamente. Los errores que se introducen es esta investigación se obtienen al comparar las fracciones molares máximas con los datos experimentales de Lucas et al. (2004), para metano: $5 \%$, para monóxido: $12 \%$, para hidrógeno: $80 \%$. 


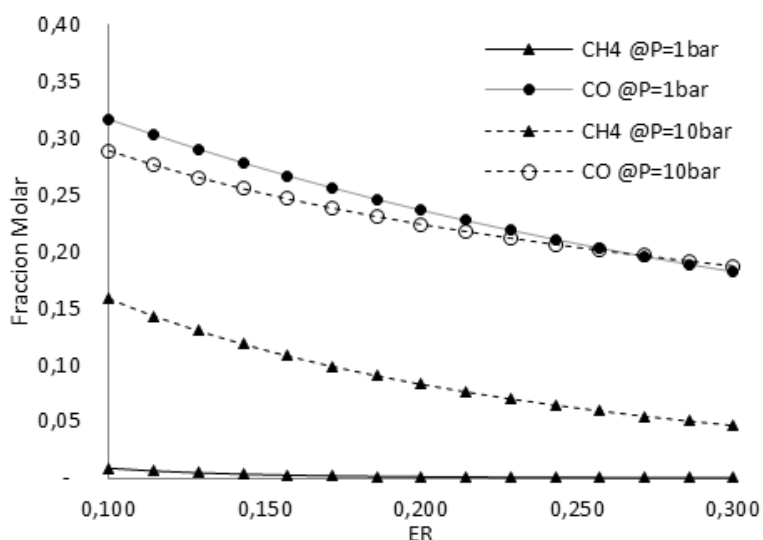

a)

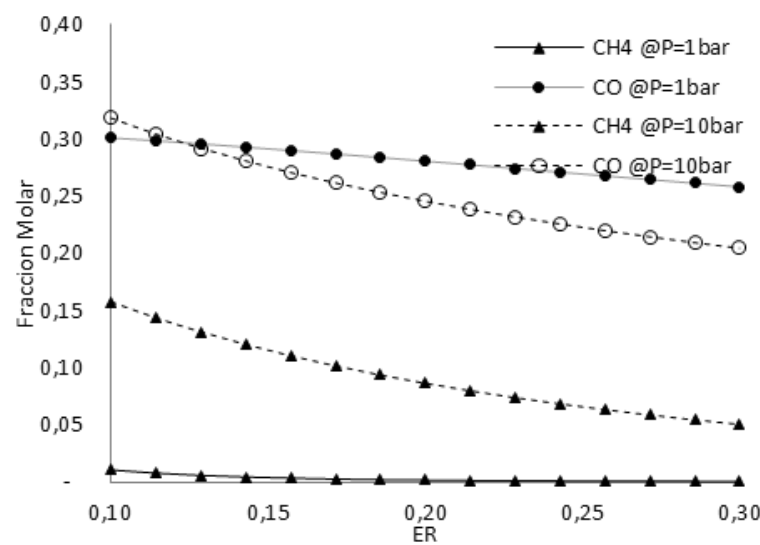

b)

Fig. 3. Variación de la composición de gas producto en función del ER a presiones de 1 bar y 10 bares a) de los residuos de de la palma africana y b) el orujo lavado de uva.

Para el efecto de la variación del equivalente ratio (ER), se evidencia en las figura $3 a$ y $3 b$, que la formación de gas producto $\mathrm{H}_{2}, \mathrm{CO}, \mathrm{CH}_{4}$ disminuye de manera significativa ocasionado por la mayor conversión de las reacciones en presencia de oxigeno, como consecuencia de esto el poder calorífico del gas producto disminuye junto con la eficiencia de conversión de biomasa. Al evaluar a diferentes temperaturas las eficiencias energéticas y exergética para los residuos de la palma africana y el orujo lavado de uva se nota que tanto la energía como la exergía del gas producto aumentan con las concentraciones de $\mathrm{H}_{2}, \mathrm{CO}$ y $\mathrm{CH}_{4}$, las cuales son favorecidas a alta presión y temperatura y con ello incrementando la eficiencia energética y exergética del proceso

Las figuras $4 a$ y $4 \mathrm{~b}$ muestran la variación de la eficiencia energética y exergética para las dos presiones analizadas en función de la temperatura. En la gasificación del orujo lavado de uva se observa un mayor cambio de rendimiento energético que el dado por la biomasa de residuos de palma de aceite para los mismos rangos de operación.

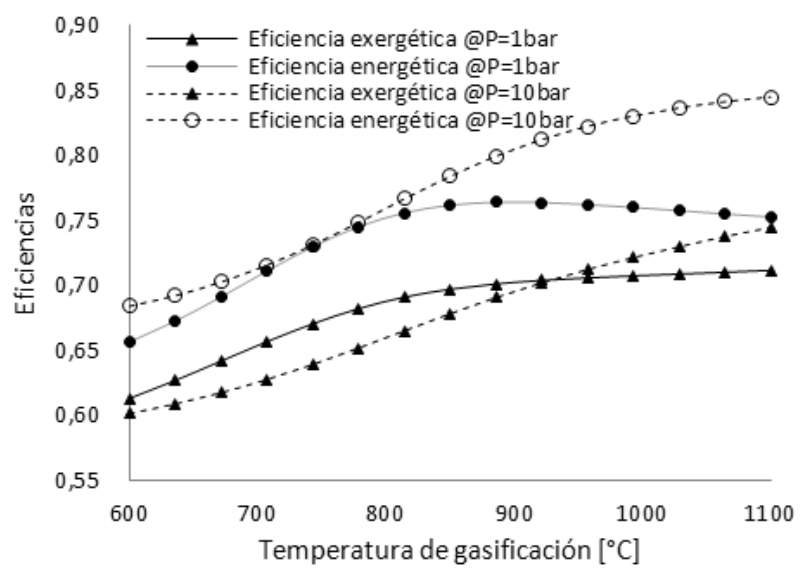

a)

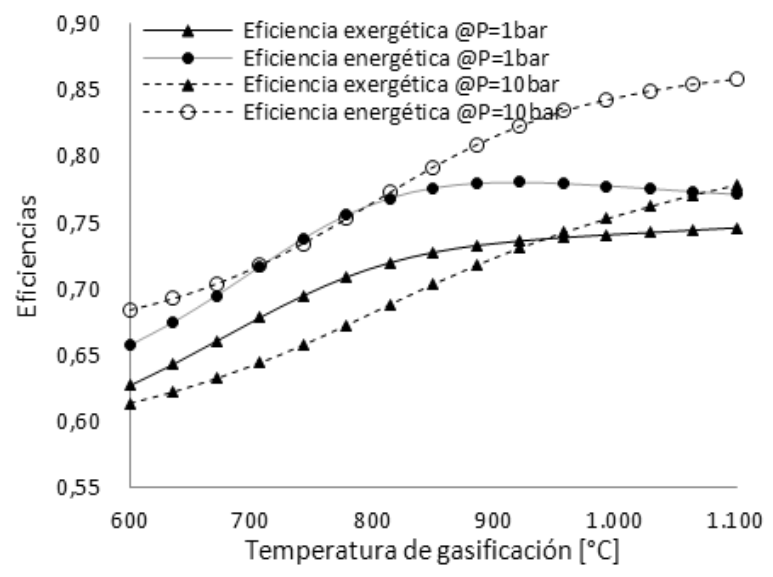

b)

Fig. 4. Eficiencias de primera ley y exergética a distintas temperaturas a presiones de 1 bar y 10 bares a) de los residuos de la palma africana y b) el orujo lavado de uva.

Las figuras $5 \mathrm{a}$ y $5 \mathrm{~b}$ muestran la variación de la eficiencia energética y exergética como función del dosado relativo. Se observa que ambas eficiencias se reducen como consecuencia de la disminución de las concentraciones de $\mathrm{H}_{2}, \mathrm{CO}$ y $\mathrm{CH}_{4}$ debido por el incremento de la relación de aire/biomasa. 


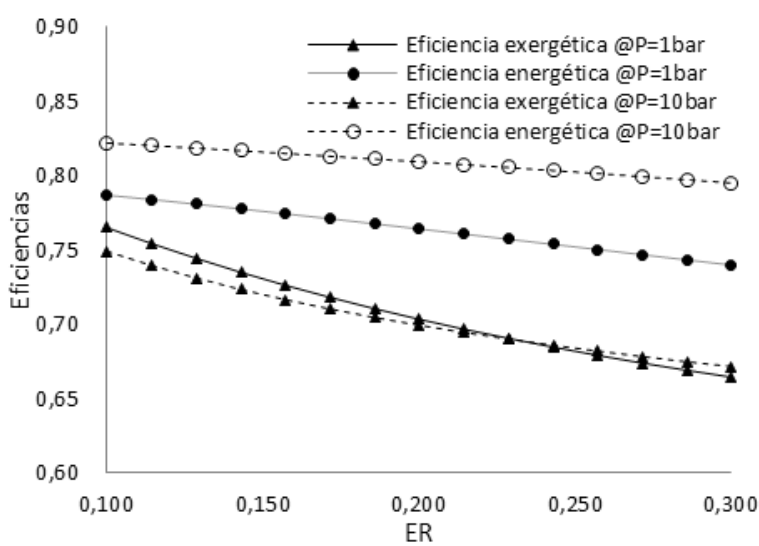

a)

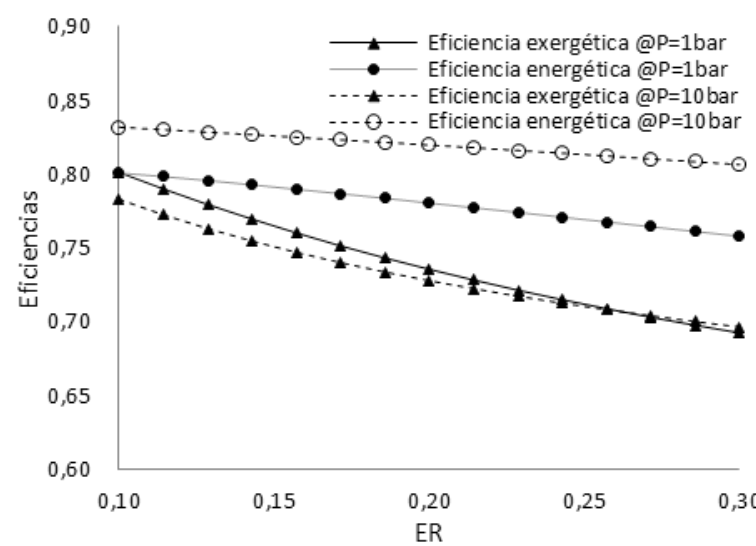

b)

Fig. 5. Eficiencias de primera ley y exergéticas a distintos valores de Equivalente Ratio a presiones de 1 y 10 bares a) de los residuos de la palma africana y b) del orujo lavado de uva.

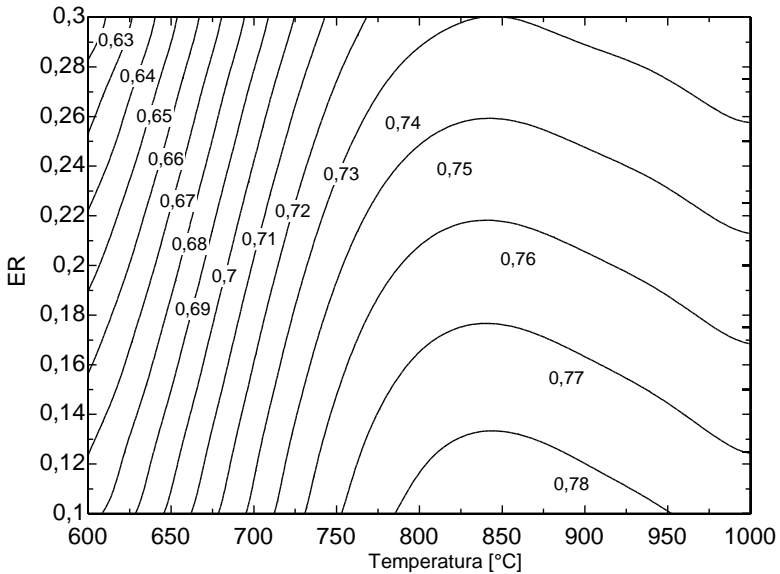

a)

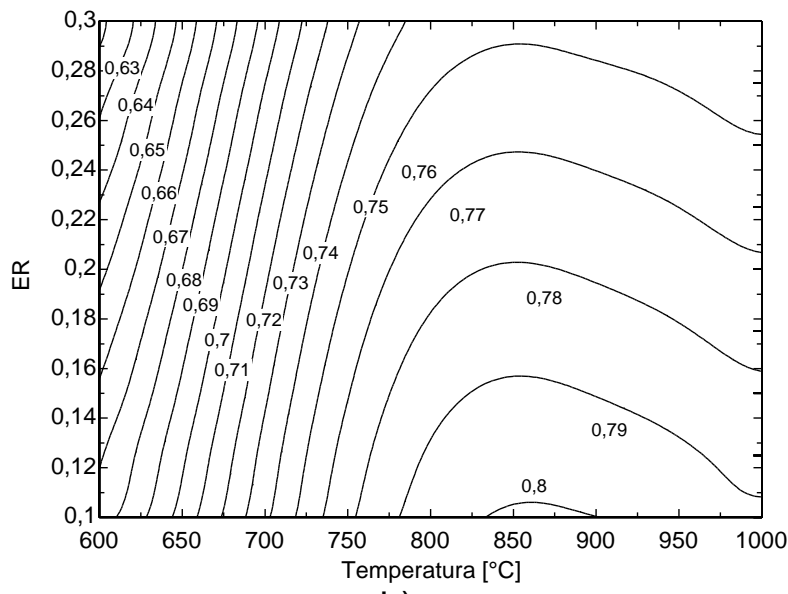

b)

Fig. 6. Superficie respuesta de la eficiencia energética a distintos valores de ER y Temperaturas a) de los residuos de la palma africana y b) del orujo lavado de uva

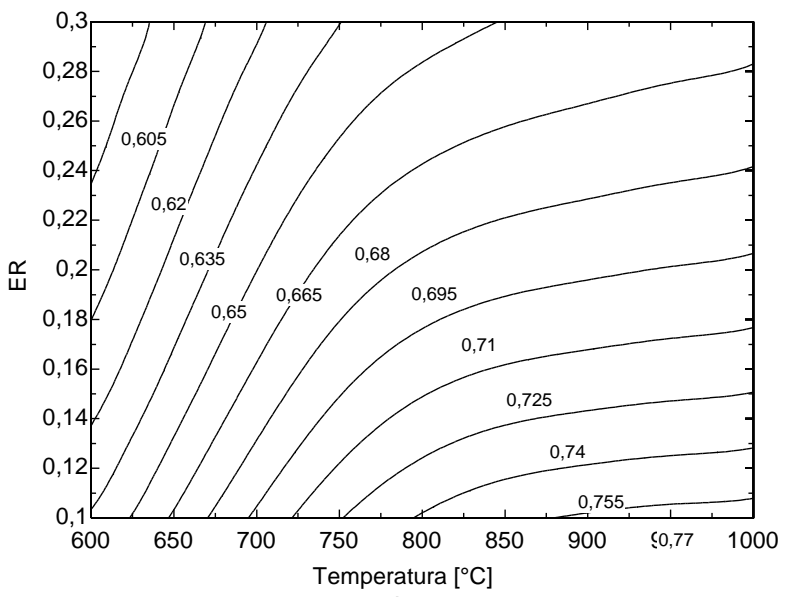

c)

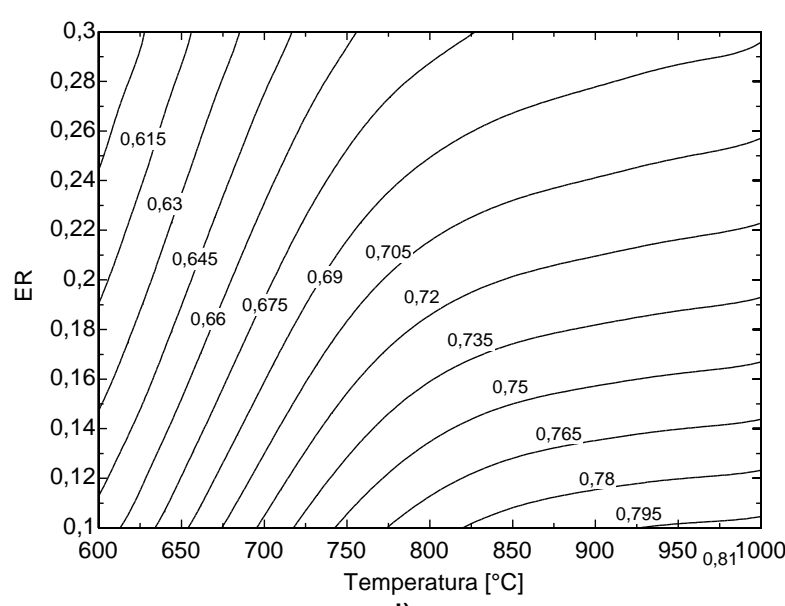

d)

Fig. 7. Superficie respuesta de la eficiencia exergética a distintos valores de ER y Temperaturas a) de los residuos de la palma africana y b) del orujo lavado de uva

Con el fin de ajustar el modelo de equilibrio termoquímico que permita parametrizar el proceso, se establecen los efectos de la temperatura y el dosado relativo mediante un análisis de superficie de respuesta a presión constante, para la eficiencia energética, exergética y el poder calorífico. Las figuras $6 \mathrm{a}, 6 \mathrm{~b}, 7 \mathrm{a}$, y $7 \mathrm{~b}$ muestran las superficies respuesta del rendimiento energético y exergético de la gasificación. Donde se observa que la biomasa de orujo lavado de uva obtiene mayores rendimientos energéticos comparados con los de los residuos de la palma africana. 
Los cambios del contenido energético del gas producto son generados por los cambios de la eficiencia en el proceso de gasificación, los cuales son gobernados por las condiciones de temperatura, presión y cantidad de aire suministrada como agente oxidante. Las figuras $8 \mathrm{a}$ y $8 \mathrm{~b}$, muestran el valor del contenido energético del gas producto por unidad de masa $[\mathrm{kJ} / \mathrm{kg}]$. Al igual que las eficiencias de primera y segunda ley, la biomasa de orujo lavado de uvas muestra un mayor contenido energética a iguales condiciones de operación que la de la gasificación de biomasa residual de palma de aceite, sin embargo la máxima cantidad de energía en la transformación termoquímica de palma puede obtenerse a menores condiciones de temperatura.

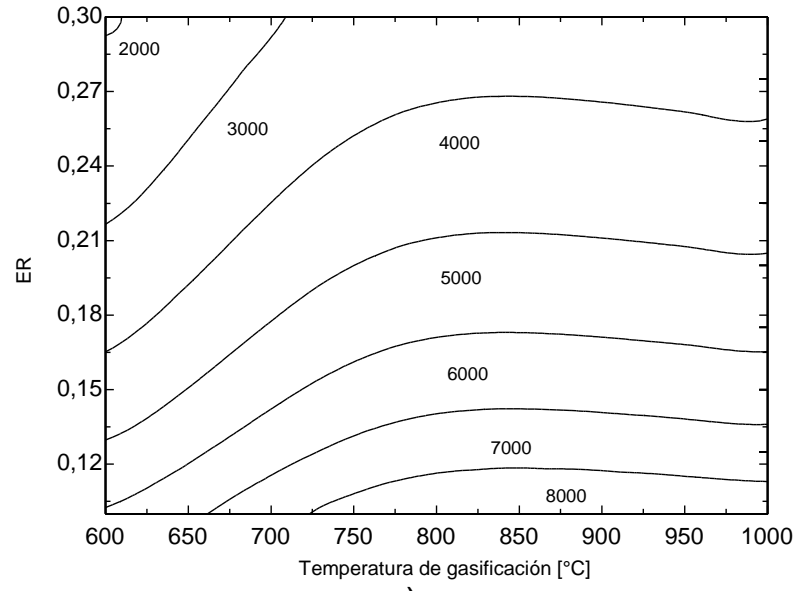

a)

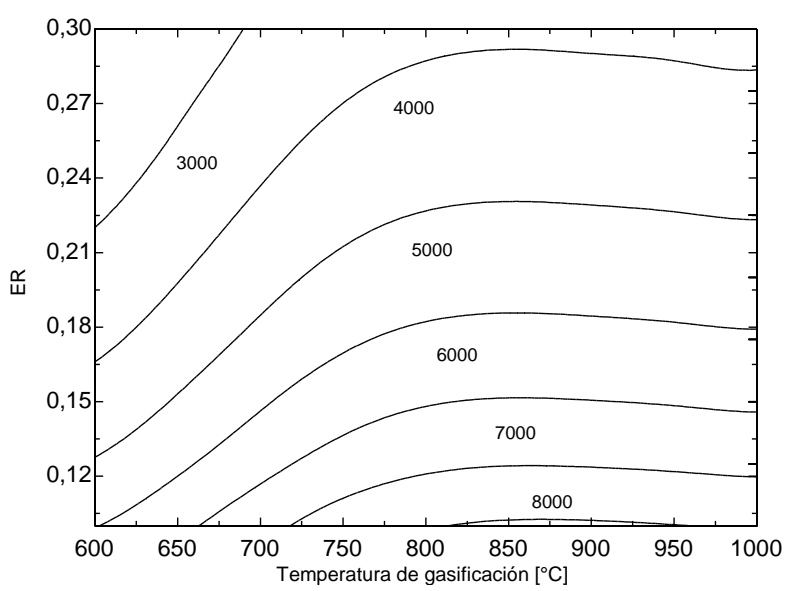

b)

Fig. 8. Superficie respuesta del poder calorífico inferior del gas producto a distintos valores de ER y Temperaturas a) del los residuos de la palma africana y b) del orujo lavado de uva.

\section{CONCLUSIONES}

Se observa una marcada influencia de la temperatura de gasificación sobre la eficiencia energética y exergética del proceso, lo cual se explica por la enorme influencia de la exergía física de los distintos elementos que componen el gas de síntesis. En este mismo orden de ideas se encontró que la composición química de la biomasa tuvo una incidencia menor sobre las eficiencias estudiadas, pues en general el análisis último es muy similar respecto al contenido de carbono $(\mathrm{C})$ Oxigeno $(\mathrm{O})$ y nitrógeno $(\mathrm{N})$, adicionalmente la cantidad de calor rechazado es variable y está ligado a la temperatura del proceso de gasificación, Con base en ello se obtuvo las superficies respuesta, composición del gas, exergía, rendimiento energético entre otros

Asumiendo la gasificación adiabática, la evaluación de la eficiencia exergética para ambas biomasas se muestra inferior a la eficiencia de primera ley en un 3\%. Este bajo valor indica que gran parte de la energía disponible en los gases es aprovechable químicamente en la combustión posterior para la generación de energía indicando una alta calidad energética en los gases y bajas irreversibilidades en el proceso.

\section{NOMENCLATURA}

$\begin{array}{ll}P_{0}: & \text { Presión atmosférica de referencia [atm] } \\ \mathrm{T}_{0}: & \text { Temperatura ambiente de referencia }\left[{ }^{\circ} \mathrm{C}\right] \\ \mathrm{g}_{\mathrm{i}}^{*}: & \text { Energía libre de Gibbs del componente } \mathrm{i}[\mathrm{kJ} / \mathrm{kmol}] \\ \boldsymbol{x}_{i} & \text { Fracción molar del componente i para el gas } \\ \mathrm{z}_{i}: & \text { Fracción másica del componente i para la composición de la biomasa } \\ \mathrm{z}_{w}: & \text { Fracción másica del agua para la composición de la biomasa } \\ \mathrm{P}_{\mathrm{gas}}: & \text { Presión del gas }[\mathrm{kPa}]\end{array}$



R: $\quad$ Constante universal de los gases $8.314472[\mathrm{~kJ} / \mathrm{kmol} \mathrm{K}]$
$\mathrm{T} \quad$ Temperatura de gasificación $\left[{ }^{\circ} \mathrm{C}\right]$
$\begin{array}{ll}\mathrm{g}_{\mathrm{i}}^{\circ} \text { : } & \text { Energía libre de Gibbs de formación del componente i a las condiciones de } \\ \text { referencia }[\mathrm{kJ} / \mathrm{kmol}]\end{array}$
$\mathrm{h}_{\mathrm{T}}$ : $\quad$ Entalpia especifica a la temperatura de gasificación $[\mathrm{kJ} / \mathrm{kmol}]$
$\mathrm{h}_{\mathrm{To}}$ : $\quad$ Entalpia especifica a la temperatura de ambiente [kJ/kmol]
$\mathrm{h}_{\mathrm{fg}}$ : $\quad$ Entalpia de evaporación para el agua a las condiciones ambiente [kJ/kmol]
$S_{i}: \quad \quad$ Entropía del componente i $\left[\mathrm{kJ} / \mathrm{kmol}^{\circ} \mathrm{C}\right]$
$S_{i}^{o}: \quad \quad$ Entropía absoluta a las condiciones de referencia $\left[\mathrm{kJ} / \mathrm{kmol}{ }^{\circ} \mathrm{C}\right]$
$n_{i}: \quad \quad$ Moles del componente i [mol]
$\boldsymbol{m}_{i}$ : $\quad$ Flujo másico del componente i [kg/s]
$\boldsymbol{M}_{i}$ : $\quad$ Masa molar del componente i $[\mathrm{kg} / \mathrm{kmol}]$
ER: $\quad$ Equivalente Ratio [\%]
$L \mathrm{HV}_{\text {biomasa }}$ : Poder calorífico inferior de la biomasa $[\mathrm{kJ} / \mathrm{kg}]$
$H \mathrm{HV}_{\text {biomasa }}$ : Poder calorífico superior de la biomasa $[\mathrm{kJ} / \mathrm{kg}]$
$L \mathrm{HV}_{\mathrm{gas}}$ : $\quad$ Poder calorífico del gas producto $[\mathrm{kJ} / \mathrm{kg}]$
$\Delta h_{i}: \quad$ Cambio de entalpia del componente i $[\mathrm{kJ} / \mathrm{kg}]$
$Q_{1}$ : $\quad$ Perdida de calor $\left[\mathrm{kJ} / \mathrm{k}_{\text {biomasa }}\right]$
$C_{c}$ : $\quad$ Poder calorífico del carbón $[\mathrm{kJ} / \mathrm{kg}]$
$\eta$ : $\quad$ Eficiencia de energética de la gasificación [\%]
$\Psi_{\mathrm{CH}}$ : $\quad$ Eficiencia exergética de la gasificación [\%]

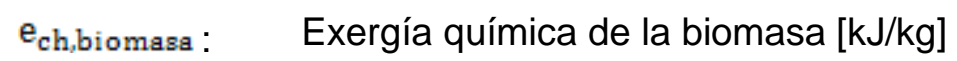
e $\quad \quad$ Exh : $\quad$ Exgía física de flujo $[\mathrm{kJ} / \mathrm{kg}]$

\section{AGRADECIMIENTOS}

Los autores agradecen a COLCIENCIAS por su apoyo mediante el contrato tesis doctoral "4392007", a la Universidad del Norte, a la Universidad Pontificia Bolivariana de Montería y al Ministerio de agricultura y desarrollo rural "2008D3614-3862".

\section{REFERENCIAS}

Abdullaha, N., y Gerhauser, H., Bio-oil derived from empty fruit bunches, Fuel, Volume 87, No 12, pp. 2606-2613 (2008).

Channiwala, S.A., Parikh P.P., A unified correlation for estimating HHV of solid, liquid and gaseous fuels. Fuel, Volume 81, pp. 1051-1063 (2002).

Chew, T., and Bhatia, S., Catalytic processes towards the production of biofuels in a palm oil, Bioresource Technology, Volume 99, pp 7911-7922 (2008).

Cujia, G., y Bula, A., Potencial Obtención de Gas de Síntesis para la Producción de metanol a partir de la Gasificación de Residuos de Palma Africana. Journal of Science and Technology of the Americas, Volume 35, No 2, pp. 106 - 112 (2010). 
Hernández, J., Aranda-Almansa, G., y Bula A., Gasification of biomass wastes in an entrained flow gasifier: Effect of the particle size and the residence time, Fuel Processing Technology, Volume 91, No 6, pp. 681-692 (2010a).

Hernández, J., Aranda-Almansa, G., San Miguel, G., y Bula, A., Gasification of Grapevine pruning waste in an entrained-flow reactor: Gas products, Energy efficiency and Gas conditioning alternatives. Global Nest Journal, Volume 12, No 2, pp. 215-227 (2010b).

Karamarkovic R., and V. Karamarkovic, Energy and exergy analysis of biomass gasification at different temperatures, Energy, Volume 35, No. 2, pp. 537-549 (2010).

Kelly T., Yong, K., Lee, T., Potential of hydrogen from oil palm biomass as a source of renewable energy worldwide, Energy Policy, Volume 35, No. 11, pp. 5692-5701 (2007).

Kotas, T. J., The exergy method of thermal plant analysis. Butterworths, London, England (1985).

Lucas, C., Szewczyka, D., Blasiaka, W., y Mochidab, S., High temperature air and steam gasificaction of densified biofuels, Biomass and Energy, Vol 27, pp. 563-575 (2004).

Mathews J., Biofuels: what a biopact between North and South could achieve, Energy Policy, Volume 35, No. 7, pp. 3550-3570 (2007).

Mendoza, J., Gomez, R. Bula, A y Corredor, L., Análisis Exergético de la Gasificación de Biomasa, $10^{\circ}$ Congreso Interamericano de Computación Aplicada a la Industria de Procesos, Girona, España, Mayo 30 - Junio 3, 2011, pp. 641-648. (2011).

Olah G., Goeppert, A., Surya Prakash, G. Beyond Oil and Gas: The Methanol Economy, Wiley$\mathrm{VCH},(2006)$.

Pazo A., Estudio del potencial de biomasa residual en Castilla-La Mancha para su explotación en centrales termoeléctricas, Universidad de Castilla-La Mancha-Universidad Rey Juan Carlos (2006).

Pérez J., Modelado unidimensional del proceso de gasificación de biomasa lignocelulósica en lechos empacados en equicorriente, Universidad de Valladolid, pp. 12, 21, 49, 96, 99, 101 (2007).

Szargut J. y Steward, F., Exergy analysis of thermal, chemical metallurgical processes. (1988). 
